\title{
The efficacy of black tea in ameliorating endothelial function is equivalent to that of green tea
}

\author{
Nicoline Jochmann ${ }^{1}$, Mario Lorenz ${ }^{1}$, Amélie von Krosigk ${ }^{1}$, Peter Martus ${ }^{2}$, Volker Böhm ${ }^{3}$, Gert Baumann ${ }^{1}$, \\ Karl Stangl ${ }^{1}$ and Verena Stangl ${ }^{1}$. \\ ${ }^{1}$ Medizinische Klinik mit Schwerpunkt Kardiologie und Angiologie, Charité - Universitätsmedizin Berlin, Campus Mitte, \\ Schumannstrasse 20-21, D-10117 Berlin, Germany \\ ${ }^{2}$ Institut für Biometrie und Klinische Epidemiologie, Charité - Universitätsmedizin Berlin, Campus Mitte, Schumannstrasse 20-21, \\ D-10117 Berlin, Germany \\ ${ }^{3}$ Institut für Ernährungswissenschaften, Friedrich-Schiller-Universität Jena, Germany \\ (Received 15 March 2007 - Revised 8 August 2007 - Accepted 24 August 2007)
}

Consumption of tea has been shown to improve endothelial function. It is assumed that catechins are the tea components responsible for these beneficial effects. In black tea, catechin concentrations are significantly lower than in green tea. The present study was designed to compare green and black tea with regard to amelioration of endothelial function. Endothelial function in response to both teas was assessed in bovine aortic endothelial cells (BAEC) and rat aortic rings. To elucidate whether these findings are also applicable to humans, flow-mediated dilation (FMD) and nitro-mediated dilation (NMD) were assessed by ultrasound in twenty-one healthy women before and $2 \mathrm{~h}$ after consumption of green and black tea ( $2 \mathrm{~h}$ of FMD and NMD), in comparison with water (control). In BAEC, green and black tea significantly increased endothelial NO synthase activity to the same extent. Similarly, both teas induced comparable endothelial-dependent vasodilation in rat aortic rings. In human subjects, ingestion of green and black tea led to significant increases in FMD: from 5.4 (SD 2.3) to 10.2 (SD 3) \% (baseline-adjusted difference (BAD) for $2 \mathrm{~h}$ of FMD, green tea $v$. water: $5.0(95 \% \mathrm{CI} 3 \cdot 0,7 \cdot 0) \% ; P<0.001)$ and from 5 (SD 2.6) to 9.1 (SD 3.6) \% (BAD for $2 \mathrm{~h}$ of FMD, black tea $v$. water: $4.4(95 \%$ CI $2.3,6.5) \% ; P<0.001)$, respectively. The increase in FMD was not significantly different between the two tea preparations (BAD for $2 \mathrm{~h}$ of FMD, green tea $v$. black tea: $0.66(95 \% \mathrm{CI}-0.76,2.09) \% ; P=0.36)$. NMD did not vary between any of the groups. In conclusion, green and black tea are equally effective in improving endothelial function.

Tea: Endothelial function: Flow-mediated dilation: Nitric oxide

Compelling evidence from epidemiological and clinical studies has established a positive correlation between the consumption of green and black tea and protection against atherosclerosis and $\mathrm{CVD}^{1-4}$. In addition to the antioxidative, anti-inflammatory, anti-proliferative and anti-thrombotic properties of polyphenols contained in tea, favourable effects on endothelial function are the main underlying mechanisms suggested as being involved in the prevention of CHD by tea consumption ${ }^{5,6}$. Evidence is accumulating that catechins, the main polyphenolic compounds in green tea, are the substances responsible for these beneficial effects. Accordingly, we and others have found that catechins, particularly epigallocatechin-3-gallate (EGCG), evoke endothelial-dependent vasodilation via rapid activation of endothelial NO synthase (eNOS $)^{7,8}$.

Both green and black teas contain catechins. In black tea, however, catechin concentrations are significantly lower than in green tea. This is ascribed to the manufacturing process, which either prevents or allows tea polyphenols in the leaves to be oxidised. Whereas in green tea the intention is to avoid oxidation of polyphenols, black tea is manufactured by promoting enzymic oxi- dation (fermentation). During this process, catechins are oxidised to theaflavins and thearubigins. As a result, catechins constitute about $80-90 \%$ of total flavonoids in green tea, whereas catechin content in black tea is only $20-50 \%$ or even lower ${ }^{9}$. Tea is the major beverage after water consumed in the world. Since green tea is mainly consumed in East Asia and black tea is preferred in the Western world, a question of much interest is whether green tea is superior to black tea regarding beneficial effects on endothelial function ${ }^{10}$. The aim of the present study was therefore to compare the effects of green and black tea on the production of NO in endothelial cells, on vasorelaxation in rat aortic ring preparations, and on flow-mediated dilation (FMD) in human subjects.

\section{Methods \\ Measurement of endothelial nitric oxide synthase activity and phosphorylation}

Bovine aortic endothelial cells were maintained and incubated as recently described ${ }^{11}$. Subsequently, bovine aortic endothelial 
cells were treated with the indicated doses of green or black tea for $15 \mathrm{~min}$. eNOS activity was assessed by the formation of $\mathrm{L}-\left[{ }^{3} \mathrm{H}\right]$ citrulline from $\mathrm{L}-\left[{ }^{3} \mathrm{H}\right]$ arginine after separation of the amino acids by cation exchange chromatography as described previously $^{8}$. Briefly, stimulation was initiated by addition of the tea preparations, $10 \mu \mathrm{M}$-cold $\mathrm{L}$-arginine, and $\mathrm{L}-\left[{ }^{3} \mathrm{H}\right]$ arginine $(111 \mathrm{GBq}(3 \mu \mathrm{Ci}) / \mathrm{ml})$. After $15 \mathrm{~min}$, the reaction was terminated with ice-cold stop solution containing $5 \mathrm{mM}$-L-arginine and 4 mM-EDTA. Cells were denatured with $96 \%$ ethanol and, after evaporation, the soluble cellular components were extracted with $20 \mathrm{mM}-\mathrm{HEPES}-\mathrm{Na}(\mathrm{pH} 5 \cdot 5)$. L- $\left[{ }^{3} \mathrm{H}\right]$ citrulline was separated from $\mathrm{L}-\left[{ }^{3} \mathrm{H}\right]$ arginine by Dowex chromatography, and $\mathrm{L}-\left[{ }^{3} \mathrm{H}\right]$ citrulline formation was quantified by liquid scintillation counting. For Western blots, cells were treated with the two types of tea, or water as control, washed twice with PBS, and lysed in buffer containing (mM): HEPES ( $\mathrm{pH}$ 7.9), 20; $\mathrm{NaCl}, 100 ; \mathrm{Na}_{3} \mathrm{VO}_{4}, 1$; sodium pyrophosphate, 4; EDTA, 10; phenylmethylsulfonyl fluoride, 1 ; NaF, 10; okadaic acid, $0 \cdot 1$; Triton $\mathrm{X}-100,1 \%$. Total protein $(15 \mu \mathrm{g}$ per lane) was subjected to SDS-PAGE, and membranes were probed with antieNOS from BD Transduction Laboratories (Lexington, KY, USA) and with anti-phospho-eNOS (Ser1179) from Cell Signaling Technologies (Beverly, MA, USA). Bands were visualised by using either BCIP (5-bromo-4-chloro-3-indolyl phosphate, p-toluidine salt) and nitro blue tetrazolium (Sigma, Deisenhofen, Germany), or the enhanced chemiluminescence detection system (Amersham, Freiburg, Germany).

\section{Vasorelaxation studies}

Thoracic aortas from male Wistar rats were rapidly excised, cleaned of connective tissue, and cut into rings 2 to $3 \mathrm{~mm}$ in length for organ-chamber experiments. The rings were prepared as recently described ${ }^{8}$. Following equilibration and submaximal precontraction with phenylephrine $(0.05 \mu \mathrm{M})$, relaxation to cumulative doses $(5-50 \mu \mathrm{l})$ of black and green tea was performed. Selected studies were conducted in rings treated with the NOS inhibitor $N$-nitro-L-arginine methyl ester (1 mM) before phenylephrine exposure. Vasorelaxation is expressed as percentage of precontraction with phenylephrine.

\section{Endothelial function in human subjects}

Endothelial function was measured by high-resolution vascular ultrasound (Sonoline Antares; Siemens, Erlangen, Germany) as recently described ${ }^{11}$. Briefly, endothelium-dependent FMD was assessed by measuring the change in brachial artery diameter after reactive hyperaemia for $2 \mathrm{~min}$, according to established guidelines ${ }^{12,13}$. Endothelium-independent nitro-mediated dilation (NMD) was measured after sublingual application of nitroglycerine spray $(0.4 \mathrm{mg})$ for $6 \mathrm{~min}$. FMD and NMD were defined as the maximum percentage change in brachial artery diameter compared with baseline measurement. Analyses of diameter changes were conducted offline (Tom Tec Imaging Systems, Unterschleissheim, Germany) by two different investigators blinded to subject treatment.

\section{Study design}

As part of our research, we recently showed that addition of milk to black tea inhibits the vascular effects of black tea alone ${ }^{11}$. In the present analysis we investigated the effects of green and black tea on FMD in human subjects in a direct comparison of the two types of tea. Study subjects were recruited by press advertisements. None of the participants had taken medication for at least 3 months before entering the study. Subjects with cardiovascular risk factors such as high blood cholesterol, diabetes, arterial hypertension and obesity were excluded. The participants were asked not to drink tea 4 weeks before and during the study. Study subjects were required to make three clinical visits, at least $3 \mathrm{~d}$ apart, and at the same time of the day after fasting overnight. Each subject consumed either $500 \mathrm{ml}$ boiled water, freshly brewed black tea or green tea in a cross-over study design. A quantity of $5 \mathrm{~g}$ tea leaves (Darjeeling black or Darjeeling green tea; King's Teagarden, Berlin, Germany) were brewed for $3 \mathrm{~min}$ with $500 \mathrm{ml}$ boiled water. FMD and NMD were measured before and $2 \mathrm{~h}$ after (i.e. $2 \mathrm{~h}$ of FMD and NMD) consumption of the beverages. The participants had a standardised breakfast during consumption of the beverage. The study was approved by the Charité University Hospital Ethics Committee, and participants provided their written informed consent.

\section{Analysis of tea components}

Tea was prepared as described in the Study design. The concentrations of individual tea substances in brewed tea were determined as described, with slight modifications ${ }^{14}$. In brief, tea samples were diluted with $10 \%$ acetonitrile containing EDTA $(500 \mu \mathrm{g} / \mathrm{ml})$ and ascorbic acid. The samples were analysed by HPLC. The HPLC detection system consisted of an Agilent 1100 (Agilent Technology, San Diego, CA, USA) with a binary pump, a thermostated autosampler, a column oven, a photodiode array detector, and a data system with Agilent 1100 ChemStation software. The column was eluted at $35^{\circ} \mathrm{C}$ with a binary gradient of $100 \%$ solution A (9\% acetonitrile, $2 \%$ acetic acid, containing EDTA $(20 \mu \mathrm{g} / \mathrm{ml}))$ for $10 \mathrm{~min}$, $68 \%$ solution A and $32 \%$ of solution B ( $80 \%$ acetonitrile, $2 \%$ acetic acid, containing EDTA $(20 \mu \mathrm{g} / \mathrm{ml}))$ for $10 \mathrm{~min}$ at a flow rate of $1.0 \mathrm{ml} / \mathrm{min}$. The eluent was monitored at $278 \mathrm{~nm}$. The signals were verified by using UV spectra (diode array detector) and comparisons of the retention times with reference compounds. Quantification was carried out using the relative response factor concept of ISO $14505-2^{14}$.

\section{Statistical analysis}

Goodness of fit for normal distribution was examined by determining skewness and kurtosis. A general linear model was applied to compare the three beverages (water, black tea, and green tea). Baseline was included as a continuous covariate, and the beverages and time (as a nuisance factor) were coded by dummy variables. In cases of overall significance, pairwise tests were performed without further adjustment for multiple testing (closed testing procedure) ${ }^{15}$. Adjustment of the correlation of measurements from the same subjects took place by application of generalised estimating equations ${ }^{16}$. No time effects or time $v$. treatment interactions were found. All statistical tests were two-sided (level of significance $=0.05)$. We performed statistical analysis by using SPSS (release 12.0.1; SPSS Inc., Chicago, IL, USA), except for generalised estimating equations analysis, in 
which we applied SAS (release 9.1; SAS Institute Inc., Cary, NC, USA).

\section{Results}

\section{Analysis of tea components}

We measured the concentrations of various tea compounds, including the catechins, in both green tea and black tea preparations. The concentrations of single tea compounds are shown in Table 1 . The overall catechin concentration in black tea was about half that in green tea. The concentrations of EGCG - one of the most potent catechins - were $30 \%$ lower in black tea than in green tea.

Measurement of endothelial nitric oxide synthase activity in endothelial cells

To clarify whether eNOS activation is one of the underlying mechanisms by which both teas enhance endothelial function, we incubated bovine aortic endothelial cells with increasing concentrations of green or black tea, and measured eNOS activity in intact cells. As shown in Fig. 1(a), incubation of cells with varying amounts of either green or black tea activated eNOS dose dependently. There was no difference in the magnitude of the increase in eNOS activity after treatment of cells with green or black tea. The final concentration of total catechins after addition of $100 \mu \mathrm{l}$ of green and black tea to cells was 63.2 and $35 \mu \mathrm{M}$, respectively. Likewise, both green and black tea preparations induced comparable levels of eNOS phosphorylation (Fig. 1(b)).

\section{Tea-induced vasorelaxation of rat aortic rings}

To compare the effects of green and black tea on vasoreactivity, we exposed phenylephrine-precontracted rat aortic rings to cumulative doses of the respective tea preparation. Both green and black tea (n 18) induced pronounced, dose-dependent vasorelaxation in the aortic rings (Fig. 1(c)). In aortic rings, the final concentration of total catechins after addition of $10 \mu \mathrm{l}$ of green and black tea was 1 and $0.56 \mu \mathrm{M}$, respectively. The degree of vasodilation evoked by green and black tea was virtually identical. Pretreatment of rat aortic rings with the NOS-inhibitor N-nitro-L-arginine methyl ester completely prevented tea-induced vasodilation, indicating that relaxations of rat aortic rings induced by green or black tea are due to

Table 1. Concentrations of individual tea components $(\mu \mathrm{M})$

\begin{tabular}{lcc}
\hline & Darjeeling green & Darjeeling black \\
\hline Caffeine & 814 & 1034 \\
Gallic acid & 30 & 91 \\
Catechin & 30 & 0 \\
Gallocatechin & 52 & 0 \\
Epicatechin & 79 & 50 \\
Epigallocatechin & 257 & 70 \\
Epicatechin gallate & 130 & 116 \\
Epigallocatechin-3-gallate & 464 & 324 \\
Total catechins & 1012 & 560 \\
\hline
\end{tabular}

(a)

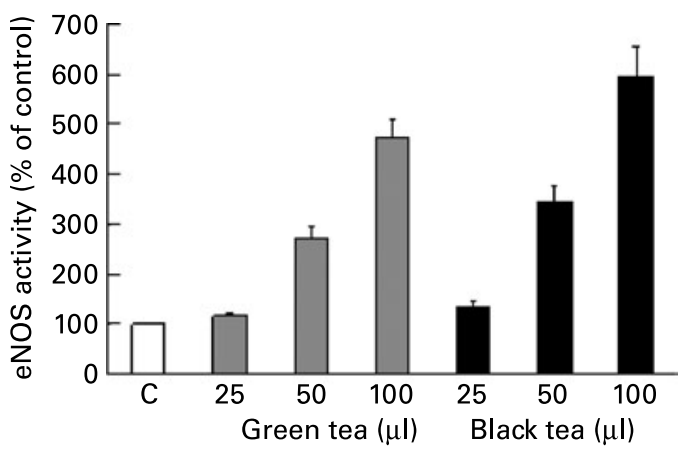

(b)

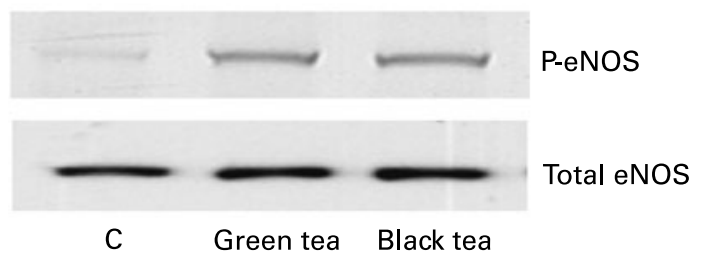

(c)

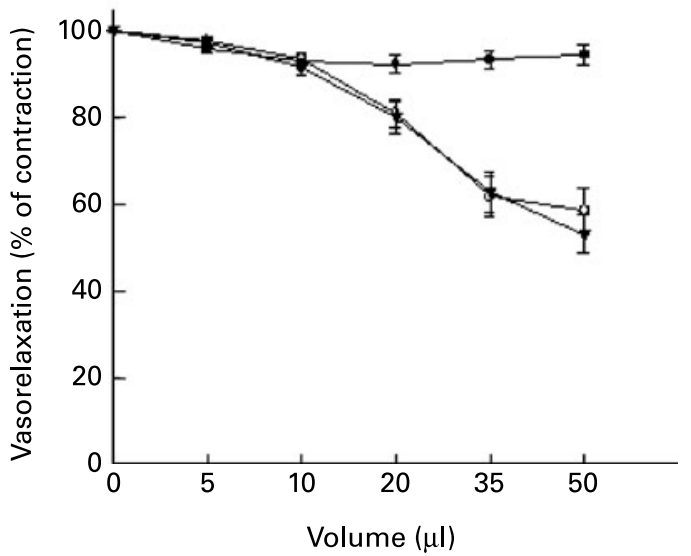

Fig. 1. Impact of green and black tea preparations on NO production in endothelial cells and on vasorelaxation in aortic rings. (a) Cells were treated for 15 min with the indicated amounts of green or black tea, or with water (control; $\mathrm{C}$ ), and endothelial NO synthase (eNOS) activity was measured. Values are means $(n 5)$, with standard errors represented by vertical bars. (b) Endothelial cells were treated for $15 \mathrm{~min}$ with $100 \mu \mathrm{l}$ of the indicated tea preparations. The Western blot showing the phosphorylation of eNOS (P-eNOS) was performed with an antibody specific to the eNOS $\operatorname{Ser}^{1179}$ phosphorylation site. Total eNOS served as the control for equal loading. The Western blot is representative of three independent experiments. (c) Precontracted rat aortic rings were treated with cumulative doses of either water (control; - - -; n 16), green tea $\left(--_{-} ; n 18\right)$ or black tea $(-\nabla-; n 18)$, and vasorelaxation was determined. Vasorelaxation is expressed as percentage of contraction. Values are means, with standard errors represented by vertical bars.

generation of NO, and are hence endothelial dependent. The vasodilating effect of tea occurred rapidly, within minutes.

\section{Endothelial function in human subjects}

A total of twenty-one healthy postmenopausal women completed the study. Baseline characteristics of the study group are shown in Table 2. Endothelial function was assessed by measuring FMD of the forearm brachial artery, before and $2 \mathrm{~h}$ after ingestion either of $500 \mathrm{ml}$ water (control), black 
Table 2. Baseline characteristics of the study population (Mean values and standard deviations for twenty-one subjects)

\begin{tabular}{|c|c|c|}
\hline Characteristic & Mean & SD \\
\hline Age (years) & $58 \cdot 7$ & 4.5 \\
\hline Systolic blood pressure (mmHg) & 118.5 & 13.7 \\
\hline Diastolic blood pressure $(\mathrm{mmHg})$ & $72 \cdot 1$ & 8.0 \\
\hline BMI $\left(\mathrm{kg} / \mathrm{m}^{2}\right)$ & $23 \cdot 1$ & 1.7 \\
\hline $\mathrm{HbA} 1_{\mathrm{c}}(\%)$ & $5 \cdot 8$ & 0.9 \\
\hline Homocysteine $(\mu \mathrm{mol} / \mathrm{l})$ & $10 \cdot 2$ & $2 \cdot 1$ \\
\hline C-reactive protein (mg/l) & 1 & 1 \\
\hline Total cholesterol $(\mathrm{mmol} / \mathrm{l})$ & $5 \cdot 3$ & 0.9 \\
\hline HDL-cholesterol (mmol/l) & 1.9 & 0.3 \\
\hline LDL-cholesterol (mmol//) & $3 \cdot 1$ & 0.9 \\
\hline TAG $(\mathrm{mmol} / \mathrm{l})$ & 0.84 & 0.30 \\
\hline Lipoprotein(a) (mg/l) & 100 & 87 \\
\hline
\end{tabular}

tea, or green tea in a cross-over study. All results of the intervention study are presented in detail in Tables 3 and 4. Briefly, ingestion of green and black tea led to significant increase of FMD: from 5.4 (SD 2.3) to 10.2 (SD 3) \% (baseline-adjusted difference for $2 \mathrm{~h}$ of FMD, green tea $v$. water: 5.0 (95\% CI $3.0,7.0) \% ; P<0.001$ ), and from 5 (SD 2.6) to 9.1 (SD 3.6) $\%$ (baseline-adjusted difference for $2 \mathrm{~h}$ of FMD, black tea $v$. water: $4.4(95 \%$ CI $2.3,6.5) \% ; P<0.001)$, respectively (Fig. 2). The increase in FMD was not significantly different between the two tea preparations (baseline-adjusted difference for $2 \mathrm{~h}$ of FMD, green tea $v$. black tea: 0.66 (95\% CI -0.76, $2 \cdot 09) \% ; P=0 \cdot 36)$. Water showed no effect on FMD. Endothelial-independent NMD was not significantly different after consumption of the three beverages.

\section{Discussion}

The present study is the first to compare the effects of green and black tea on endothelial function in vitro and in vivo. In both rat aortic rings and in healthy women, green and black tea significantly enhanced endothelial function in a comparable manner. The amount of tea-induced increase of eNOS activity in endothelial cells was furthermore not different after incubation of the cells with green or black tea. In the present study, we used freshly brewed tea (rather than tea extracts) for our in vivo as well as in vitro studies, to mimic real-life conditions. Although, as expected, the catechin content was much lower in black tea (Table 1), the efficacy of this fermented tea preparation in terms of amelioration of endothelial function was identical to green tea.

Several clinical studies have described beneficial effects of both green and black tea on endothelial function ${ }^{11,17-19}$. We recently showed that addition of milk to black tea completely prevented the improvement of endothelial function by black tea alone in human subjects. In addition to these in vivo results, we were able to demonstrate a selective decrease in concentrations of catechins in tea with milk compared with tea without milk - findings that suggest an important role of catechins in black tea for amelioration of endothelial function ${ }^{11}$.

The underlying molecular mechanisms for favourable teainduced effects on endothelial function are multifactorial; they involve direct effects on the NO system, as well as antioxidative effects associated with an increase in NO availability. Although information is limited concerning the biological activities of theaflavins, and especially of thearubigins - the heterogeneous oxidation products that are the main polyphenols in black tea - there is increasing evidence that they are effective antioxidants ${ }^{20,21}$. However, data are not uniform with regard to the antioxidative capacity of theaflavins as compared with catechins. Lee et al. reported that the antioxidative capacity per serving of green tea is much higher than that of black tea in commercial tea products ${ }^{22}$. Some authors found that the antioxidant effects of theaflavins in black tea are equal to that of catechins in green tea ${ }^{23}$. Yoshida et al. have shown that theaflavin digallate had a higher inhibitory effect on LDL oxidation in macrophages in vitro when compared with EGCG. The authors postulated that the higher antioxidative capacity of theaflavins is due to their greater number of hydroxyl $(\mathrm{OH})$ groups, since theaflavins represent polymers of catechins ${ }^{20}$. It is assumed that the degree of hydroxylation is positively correlated with their antioxidant efficiency ${ }^{24}$. Antioxidative activities of black tea thearubigins on lipid peroxidation have likewise been reported ${ }^{25}$. In conclusion, these data suggest that the antioxidative effects of green and black tea may differ depending on the polyphenol composition.

Beyond the antioxidant properties of catechins and their oxidised products theaflavins and thearubigins, direct effects on the NO system may also contribute to improvement of endothelial function after green and black tea consumption. We have recently shown that EGCG, the main catechin in

Table 3. Flow-mediated dilation (FMD) and nitro-mediated dilation (NMD) before and $2 \mathrm{~h}$ after consumption of water, green tea and black tea (Mean values and standard deviations for twenty-one subjects)

\begin{tabular}{|c|c|c|c|c|c|c|c|c|c|c|c|c|}
\hline & \multicolumn{2}{|c|}{ Water (basal) } & \multicolumn{2}{|c|}{ Water $(2 \mathrm{~h})$} & \multicolumn{2}{|c|}{$\begin{array}{c}\text { Green tea } \\
\text { (basal) }\end{array}$} & \multicolumn{2}{|c|}{ Green tea $(2 \mathrm{~h})$} & \multicolumn{2}{|c|}{$\begin{array}{c}\text { Black tea } \\
\text { (basal) }\end{array}$} & \multicolumn{2}{|c|}{ Black tea $(2 \mathrm{~h})$} \\
\hline & Mean & SD & Mean & SD & Mean & SD & Mean & SD & Mean & SD & Mean & SD \\
\hline $\mathrm{FMD}_{\text {base }}(\mathrm{mm})$ & 3.07 & 0.26 & $3 \cdot 10$ & 0.28 & $3 \cdot 10$ & 0.25 & $3 \cdot 10$ & 0.25 & 3.07 & 0.28 & 3.08 & 0.29 \\
\hline$F M D_{\max }(\mathrm{mm})$ & $3 \cdot 24$ & 0.28 & $3 \cdot 30$ & 0.29 & $3 \cdot 26$ & 0.24 & 3.42 & 0.27 & $3 \cdot 22$ & 0.27 & 3.35 & 0.29 \\
\hline$F M D_{\max }(\%)$ & $5 \cdot 5$ & 3.0 & 6.4 & $2 \cdot 8$ & 5.4 & $2 \cdot 3$ & $10 \cdot 2$ & $3 \cdot 0$ & $5 \cdot 0$ & $2 \cdot 6$ & 9.1 & $3 \cdot 6$ \\
\hline $\mathrm{NMD}_{\text {base }}(\mathrm{mm})$ & $3 \cdot 10$ & 0.28 & $3 \cdot 12$ & 0.27 & $3 \cdot 12$ & 0.26 & $3 \cdot 12$ & 0.26 & $3 \cdot 10$ & 0.27 & $3 \cdot 11$ & 0.29 \\
\hline $\mathrm{NMD}_{\max }(\mathrm{mm})$ & 3.71 & 0.28 & 3.78 & 0.31 & 3.72 & 0.31 & 3.80 & 0.32 & 3.72 & 0.30 & 3.78 & 0.33 \\
\hline $\mathrm{NMD}_{\max }(\%)$ & $20 \cdot 2$ & 4.5 & $21 \cdot 0$ & 4.6 & $19 \cdot 1$ & $5 \cdot 2$ & 21.4 & $4 \cdot 1$ & $20 \cdot 2$ & $5 \cdot 8$ & $21 \cdot 7$ & $4 \cdot 1$ \\
\hline
\end{tabular}

FMD base, diameter of brachial artery before hyperaemic stimulus; $F M D_{\max }$, maximum dilation of brachial artery after hyperaemic stimulus, shown in mm and as maximum percentage change in brachial artery diameter compared with $\mathrm{FMD}_{\text {base }}$; $\mathrm{NMD}_{\text {base }}$, diameter of brachial artery before application of $0.4 \mathrm{mg}$ nitroglycerine; $\mathrm{NMD}_{\text {max }}$, maximum dilation of brachial artery after application of $0.4 \mathrm{mg}$ nitroglycerine, shown in $\mathrm{mm}$ and as maximum percentage change in brachial artery diameter compared with $\mathrm{NMD}$ base. 
Table 4. Baseline-adjusted differences for $2 \mathrm{~h}$ of flow-mediated dilation (FMD) $2 \mathrm{~h}$ after consumption of green tea and black tea (Percentages and $95 \%$ confidence intervals for twenty-one subjects)

\begin{tabular}{llc}
\hline & \multicolumn{2}{c}{ Baseline-adjusted difference (\%) } \\
\cline { 2 - 3 } & FMD & $95 \% \mathrm{Cl}$ \\
\hline Green tea $v$. water & $5 \cdot 0^{*}$ & $3 \cdot 0,7 \cdot 0$ \\
Black tea $v$. water & $4 \cdot 4^{*}$ & $2 \cdot 3,6 \cdot 5$ \\
Green tea $v$. black tea & 0.66 & $-0 \cdot 76,2 \cdot 09$ \\
\hline
\end{tabular}

${ }^{*} P<0.001$.

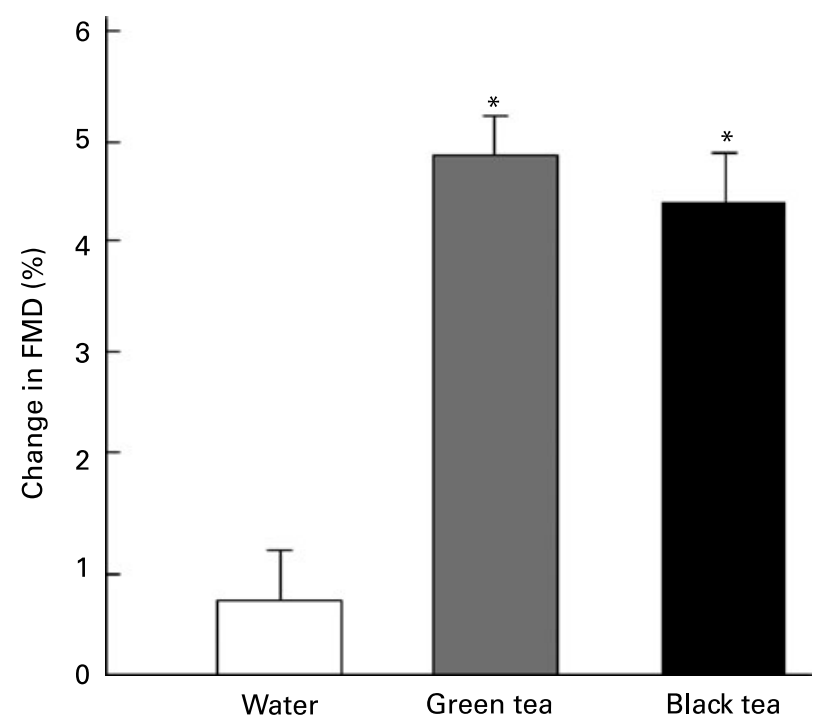

Fig. 2. Changes in flow-mediated dilation (FMD) (\%) after consumption of tea preparations. Volunteers consumed either $500 \mathrm{ml}$ of water, green tea, or black tea, and changes in FMD were measured. Values are means for twenty-one subjects, with standard errors represented by vertical bars. * Mean value was significantly higher than that for water $(P<0.001)$.

green tea, acts as a natural eNOS activator by directly enhancing eNOS activity ${ }^{8}$. In a similar manner, black tea polyphenols stimulate eNOS activity through activation of oestrogen receptor $\alpha$ in vascular endothelial cells ${ }^{26}$. In corroboration of these data, we found a comparable increase in eNOS activity by green and black tea in the present study.

At present, there are few data directly comparing the efficacy of green and black tea on cardiovascular effects. Here we show for the first time that green and black teas are equally effective in ameliorating endothelial function. Accordingly, drinking black tea may result in beneficial cardiovascular health effects similar to those of green tea. These results suggest that other polyphenolic compounds, such as theaflavins and thearubigins generated during oxidation of catechins in the process of black tea manufacture, may compensate for the reduced catechin content in black tea.

\section{Acknowledgements}

We would like to thank Angelika Vietzke, Thomas Düsterhöft and Wanda Michaelis for their excellent technical assistance.

\section{References}

1. Hertog MG, Feskens EJ, Hollman PC, Katan MB \& Kromhout D (1993) Dietary antioxidant flavonoids and risk of coronary heart disease: the Zutphen Elderly Study. Lancet 342, $1007-1011$

2. Hertog MG, Kromhout D, Aravanis C, et al. (1995) Flavonoid intake and long-term risk of coronary heart disease and cancer in the Seven Countries Study. Arch Intern Med 155, 381-386.

3. Geleijnse JM, Launer LJ, Hofman A, Pols HA \& Witteman JC (1999) Tea flavonoids may protect against atherosclerosis: the Rotterdam Study. Arch Intern Med 159, 2170-2174.

4. Sesso HD, Gaziano JM, Buring JE \& Hennekens CH (1999) Coffee and tea intake and the risk of myocardial infarction. Am J Epidemiol 149, 162-167.

5. Sano J, Inami S, Seimiya K, Ohba T, Sakai S, Takano T \& Mizuno K (2004) Effects of green tea intake on the development of coronary artery disease. Circ $J \mathbf{6 8}, 665-670$.

6. Nakachi K, Matsuyama S, Miyake S, Suganuma M \& Imai K (2000) Preventive effects of drinking green tea on cancer and cardiovascular disease: epidemiological evidence for multiple targeting prevention. Biofactors 13, 49-54.

7. Huang Y, Chan NWK, Lau CW, Yao XQ, Chan FL \& Chen ZY (1999) Involvement of endothelium/nitric oxide in vasorelaxation induced by purified green tea (-)epicatechin. Biochem Biophys Acta 1427, 322-328.

8. Lorenz M, Wessler S, Follmann E, Michaelis W, Dusterhoft T, Baumann G, Stangl K \& Stangl V (2004) A constituent of green tea, epigallocatechin-3-gallate, activates endothelial nitric oxide synthase by a phophatidylinositol-3-OH-kinase-, cAMP-dependent protein kinase-, and Akt-dependent pathway and leads to endothelial-dependent vasorelaxation. J Biol Chem 279, 6190-6195.

9. Balentine DA, Wiseman SA \& Bouwens LC (1997) The chemistry of tea flavonoids. Crit Rev Food Sci Nutr 37, 693-704.

10. Robertson A (1992) The chemistry and biochemistry of black tea production - the non-volatiles. In Tea: Cultivation to Consumption, chapter 17, pp. 555-601. [K Willson and M Clifford, editors]. London: Chapman and Hall.

11. Lorenz M, Jochmann N, Krosigk A, Martus P, Baumann G, Stangl K \& Stangl V (2007) Addition of milk prevents vascular protective effects of tea. Eur Heart J 28, 219-223.

12. Corretti MC, Anderson TJ, Benjamin EJ, et al. (2002) Guidelines for the ultrasound assessment of endothelial-dependent flow-mediated vasodilation of the brachial artery: a report of the International Brachial Artery Reactivity Task Force. J Am Coll Cardiol 39, 257-265.

13. Pyke KE \& Tschakovsky ME (2005) The relationship between shear stress and flow-mediated dilation: implications for the assessment of endothelial function. $J$ Physiol 568, 357-369.

14. Anonymous (2005) Determination of Substances Characteristic of Green and Black Tea. Part 2: Content of Catechins in Green Tea. Method using High-performance Liquid Chromatography. International Standard ISO 2005; 14502-2. Geneva: International Organization for Standardization.

15. Hochberg Y \& Tamhane AC (1987) Multiple Comparison Procedures, pp. 54. New York: John Wiley and Sons.

16. Liang KY \& Zeger SL (1986) Longitudinal data analysis using generalized linear models. Biometrika 73, 13-22.

17. Duffy SJ, Keaney JF, Holbrook M, Gokce N, Swerdloff PL, Frei B \& Vita JA (2001) Short- and long-term black tea consumption reverses endothelial dysfunction in patients with coronary artery disease. Circulation 104, 151-156.

18. Nagaya $\mathrm{N}$, Yamamoto $\mathrm{H}$, Uematsu $\mathrm{M}$, Itoh $\mathrm{T}$, Nakagawa $\mathrm{K}$, Miyazawa T, Kangawa K \& Miyatake K (2004) Green tea 
reverses endothelial dysfunction in healthy smokers. Heart $\mathbf{9 0}$, $1485-1486$.

19. Hodgson JM, Puddey IB, Burke V, Watts GF \& Beilin LJ (2002) Regular ingestion of black tea improves brachial artery vasodilator function. Clin Sci 102, 195-201.

20. Yoshida H, Ishikawa T, Hosoai H, et al. (1999) Inhibitory effect of tea flavonoids on the ability of cells to oxidize low density lipoprotein. Biochem Pharm 58, 1695-1703.

21. Menet MC, Sang S, Yang CS, Ho CT \& Rosen RT (2004) Analysis of theaflavins and thearubigins from black tea extract by MALDI-TOF mass spectrometry. J Agric Food Chem 52, 2455-2461.

22. Lee KW, Lee HJ \& Lee CY (2002) Antioxidant activity of black tea $v s$. green tea. $J$ Nutr $\mathbf{1 3 2}, 785$.
23. Leung LK, Su Y, Chen R, Zhang Z, Huang Y \& Chen ZY (2001) Theaflavins in black tea and catechins in green tea are equally effective antioxidants. $J$ Nutr 131, 2248-2251.

24. Rice Evans CA, Miller NJ \& Paganga G (1996) Structure-antioxidant activity relationships of flavonids and phenolic acids. Free Radic Biol Med 20, 933-956.

25. Yoshino K, Hara Y, Sano M \& Tomita I (1994) Antioxidative effects of black tea theaflavins and thearubigin on lipid peroxidation of rat liver homogenates induced by tert-butyl hydroperoxide. Biol Pharm Bull 17, 146-149.

26. Anter E, Chen K, Shapira OM, Karas RH \& Keaney JF (2005) p38 Mitogen-activated protein kinase activates eNOS in endothelial cells by an estrogen receptor $\alpha$-dependent pathway in response to black tea polyphenols. Circ Res 96, 1072-1078. 